in approximately $50 \%$, decreases brain blood flow by directly acting on post-synaptic $\alpha 2$ receptors, decreases CSF pressure without ischemic suffering and effectively decreases brain metabolism and intracranial pressure and also able to decrease injury caused by focal ischemia. Materials and Methods: A prospective randomized study was done on 50 patients (ASA grade 1, 2) scheduled for intracranial tumor surgeries were divided into study or control group (25 each). Both group received Midazolam $(0.02 \mathrm{mg} / \mathrm{kg})$ iv, Glycopyrrolate $(0.005 \mathrm{mg} / \mathrm{kg})$ iv, Inj Fentanyl $(2 \mu \mathrm{g} / \mathrm{kg})$ iv and group D received dexmedetomidine $0.8 \mu \mathrm{g} / \mathrm{kg}$ iv in $20 \mathrm{ml}$ saline over 10 mins and group $C$ received $20 \mathrm{ml}$ Normal saline iv for same time period without dexmedetomidine in Premedication. Patients were induced with inj. Propofol (iv) after giving test drug and dose was calculated by BIS values between 40-60, followed by inj. Vecuronium $0.1 \mathrm{mg} / \mathrm{kg}$ after recording hemodynamic measurements. Patients were ventilated with 100\% oxygen for 180 seconds. Hemodynamic measurements were recorded two minutes before test drug and placebo, two minutes before and one minutes after induction and just after and one minutes, two minutes, five minutes and 10 minutes of intubation. Change in intraocular pressure with the help of schiotz tonometer is also noted during this period. The groups were compared with heart rate, systolic blood pressure, and diastolic blood pressure and mean arterial pressure, IOP and IV dose of Propofol requirement for induction. Results: Groups were well matched for their demographic data $(P>0.05)$ and preoperative vitals. Heart rate and mean arterial blood pressure decreased significantly in patients of group D (Dexmedetomidine group) compared to group $C$ (placebo group) $(P<0.05)$, and patients were more hemodynamicaly stable before induction, and at T-1, T-2, T-5, T-10 minutes after induction in group $\mathrm{D}(P$ value $<0.05)$. The total propofol requirements for induction is more in patients of group $C$ than in patients of group D $(128.6 \pm 15.5 \mathrm{mg}$ vs. $91.8 \pm 20.41 \mathrm{mg}$, $P<0.05)$. The intraocular pressure in right eye and left eye decreases significantly from pre-induction value of 21.97 $\pm 4.75 \mathrm{~mm} \mathrm{Hg}$ and $22.08 \pm 4.94 \mathrm{~mm} \mathrm{Hg}$ to $18.02 \pm 4.30$ $\mathrm{mm} \mathrm{Hg}$ at intubation and $17.78 \pm 4.15 \mathrm{~mm} \mathrm{Hg}$ one minutes after intubation in both the eyes in group $\mathrm{D}$ after the test drug. In Group C IOP at pre-induction, intubation and one minute after intubation are $20.96 \pm 3.80 \mathrm{~mm} \mathrm{Hg}, 26.55$ $\pm 4.08 \mathrm{~mm} \mathrm{Hg}$ and $25.99 \pm 3.78 \mathrm{~mm} \mathrm{Hg}$ in right eye and $21.05 \pm 3.72 \mathrm{~mm} \mathrm{Hg}, 26.38 \pm 4.14$ and $25.90 \pm 3.75 \mathrm{~mm} \mathrm{Hg}$ in left eye respectively and the difference is statistically significant between the groups. Bradycardia and hypotension incidences were higher in group D. Conclusion: Dexmedetomidine is an excellent drug when used as an adjunct to general anesthesia for attenuation of pressor response. It not only decreased the magnitude of stress response to intubation but also decreased the intraocular pressure and induction dose requirement of propofol.

\section{Role of Intravenous Magnesium sulphate in spinal surgery for hypotensive anesthesia}

\author{
M . Somani, S. Sachdev, V. Mathur, D. D. Jethava, \\ D. Jethava
}

Department of Anaesthesiology and Critical Care, Mahatma Gandhi Medical College, Jaipur, Rajasthan, India

Background: This randomized, double-blind placebo controlled study was undertaken to evaluate effect of intravenous magnesium sulphate for hypotensive anesthesia in patient for spinal surgery. Materials and Methods: Sixty patients undergoing spinal surgery were included in two parallel groups. The magnesium group $(\mathrm{M})$ received magnesium sulphate $40 \mathrm{mg} / \mathrm{kg}$ as a bolus before induction of anesthesia and $10 \mathrm{mg} / \mathrm{kg} / \mathrm{hr}$ by continuous infusion during operation through out surgery. The control group (C) as above received isotonic saline solution both as bolus and infusion at the same rate. Result: The surgical time was reduced in magnesium group [170 (40) minutes] vs. [180 (45) minutes] in control group. The anesthetic requirements (fentanyl, vecuronium, isoflurane), mean arterial pressure $(P<0.005)$ and heart rate $(P<0.005)$ were also significantly reduced. However, the anesthetic time was longer as observed thus a prolongation in emergence from anaesthesia. Conclusion: Intravenous magnesium sulphate led to a significant decrease in mean arterial pressure, heart rate, and duration of surgery. It also alters anesthetic dose requirements.

\section{Intraoperative management of cerebral arteriovenous malformations: Our experience}

\author{
Harshal Dholke, Mohan Rao, Manas Panigrahi \\ Department of Anaesthesia and ${ }^{1}$ Neurosurgery, \\ Krishna Institute of Medical Sciences. Secundrabad, \\ Andhra Pradesh, India
}

Background: To discuss the institutional experience in the intraoperative management of symptomatic patients of cerebral AVM surgery. Materials and Methods: It is a retrospective descriptive study done in patients with cerebral AVMs who were managed by surgery at KIMS hospital between Janaury 2010 to July 2013. Totally 20 patients are included in this study. Results: Of the total patients who underwent surgery there were equal no of patients who had bleeding and who did not. Of the 
20 patients 11 were ASA I, 3 ASA II, 5 ASA III, and 1 ASA IV grade. 7, 6, 3, 4 of 20 patients had blood loss $<500 \mathrm{ml}$, $500-1000 \mathrm{ml}, 1000-1500 \mathrm{ml}$, and $>1500 \mathrm{ml}$ respectively. All patients received Tranexemic acid as prophylaxis for hemorrhage in a dose of $10 \mathrm{mg} / \mathrm{kg}$ bolus followed by $3 \mathrm{mg} / \mathrm{kg}$ infusion. Two patients were not given due to cardiac issues and had more blood loss than their counterparts. All patients were shifted to SICU for 24 hours. There were 7 patients with $<500 \mathrm{ml}$ blood loss were managed by fluid resuscitation, 6 patients with $500-100 \mathrm{ml}$ were managed by fluids alone and if $\mathrm{Hb}<10$ were given $1 \mathrm{PRBC}, 3$ patients with $1000-1500 \mathrm{ml}$ loss were given 3PRBC if $\mathrm{Hb}<10: 2 \mathrm{PRBC}$ if $\mathrm{Hb}>10$ and remaining four patients with $>1500 \mathrm{ml}$ loss were managed by three or more PRBC plus three or more FFPs. Conclusion: To conclude prophylactic administration of Tranexemic acid is helpful in decreasing the amount of bleed; however, the bleed will depend on the size and duration of surgery. With the above mentioned protocol we can achieve better hemodynamics intra-operatively and better post op $\mathrm{Hb}$.

\section{Intraoperative electro-oculographic monitoring to prevent postoperative extra-ocular motor nerve dysfunction during skull base surgeries}

\section{Veena Sheshadri, B . Suparna}

Department of Anaesthesia, Vikram Hospital, Bangalore, Karnataka, India

Background: To assess the feasibility of Electro-oculographic (EOG) monitoring in preventing extra-ocular motor nerve dysfunction during skull base surgeries. Materials and Methods: Intraoperative EOG recordings were obtained using disposable needle electrodes placed on the periorbital skin and the polarity of the waves noted for interpretation. The nerves were stimulated with a monopolar electrode during skull base exposure. Triggered as well as continuous EOG responses were recorded. Results: Of the nine cases monitored, oculomotor and abducent nerves were identified in all cases, but the trochlear nerve could not be definitively identified. Five out of nine patients had no pre or postoperative extra-ocular motor nerve dysfunction. The other four patients had pre-existing deficits before surgery, which recovered completely in two, significantly in one, and was unchanged in one patient at 3-6 month follow-up. Conclusion: EOG was found to be a simple and non-invasive method of monitoring extra-ocular motor nerves intra-operatively. Further studies in larger patient populations are required to quantitate the predictive value of EOG monitoring in detecting postoperative extra-ocular motor nerve deficits.

\section{Pharmacological and non- pharmacological interventions for reducing pain on rocuronium injection - A systematic review}

\author{
Bhavna Hooda, Hemanshu Prabhakar, Gyaninder Pal \\ Singh, Zulfiqar Ali ${ }^{1}$, Mani Kalaivani ${ }^{2}$ \\ Department of Neuroanaesthesiology, ${ }^{2}$ Biostatistics, AlIMS, \\ ${ }^{1}$ Anaesthesia and Intensive Care, Govt. Medical College, \\ Srinagar, India
}

Background: Various strategies, both pharmacological and non-pharmacological, have been studied to reduce the discomfort, incidence and severity of pain on rocuronium bromide injection. The objective of this review was to assess the ability of both pharmacological and non-pharmacological interventions designed to reduce or eliminate the pain that accompanies rocuronium bromide administration, both in adults and children, whether in asleep or awake state. Materials and Methods: We searched the Cochrane Central Register of Controlled Trials (CENTRAL), MEDLINE via Ovid SP (1966 to December 2012), and EMBASE via Ovid SP (1980 to December 2012). We included all randomized controlled trials (RCTs) that compared the use of any drug or a non-pharmacological method with control patients, or those receiving no treatment to reduce the severity of pain with rocuronium injection. Our primary outcome was pain on rocuronium bromide injection measured by a pain score assessment. Our secondary outcomes were rise in heart rate and blood pressure following administration of rocuronium and adverse events related to the interventions. We used the standardized methods for conducting a systematic review as described by the Cochrane handbook for Systematic Reviews of Interventions. Two authors independently extracted details of trial methodology and outcome data from reports of all trials considered eligible for inclusion. All analyses were made on intention-to-treat basis. We used a fixed-effect model where there was no evidence of significant heterogeneity between studies and a random-effects model if heterogeneity was likely. Results: We included 66 studies with 8621 participants in the review, though most analyses were based on data from fewer participants. We noted substantial heterogeneity between trials. Therefore, it was not possible to combine the trials for the outcomes. All studies reported the primary outcome pain; few studies reported adverse events and no study reported heart rate and blood pressure changes after administration of rocuronium. Conclusion: Lidocaine was the most popular intervention drug used in 29 trials with 2256 participants. The relative risk (RR) of pain on injection if given lignocaine compared to placebo was $0.21 ; 95 \%$ CI 